\title{
Hippocampal Cannabinoid Transmission Modulates Dopamine Neuron Activity: Impact on Rewarding Memory Formation and Social Interaction
}

\author{
Michael Loureiro', Justine Renard', Jordan Zunder' and Steven R Laviolette*, I,2,3 \\ 'Department of Anatomy and Cell Biology, The Schulich School of Medicine \& Dentistry, University of Western Ontario, London, ON, Canada; \\ ${ }^{2}$ Department of Psychiatry, The Schulich School of Medicine \& Dentistry, University of Western Ontario, London, ON, Canada; ${ }^{3}$ Department of \\ Psychology, The Schulich School of Medicine \& Dentistry, University of Western Ontario, London, ON, Canada
}

\begin{abstract}
Disturbances in cannabinoid type I receptor ( $C B \mid R$ ) signaling have been linked to emotional and cognitive deficits characterizing neuropsychiatric disorders, including schizophrenia. Thus, there is growing interest in characterizing the relationship between cannabinoid transmission, emotional processing, and dopamine (DA)-dependent behavioral deficits. The CBIR is highly expressed in the mammalian nervous system, particularly in the hippocampus. Activation of the ventral hippocampal subregion (vHipp) is known to increase both the activity of DAergic neurons located in the ventral tegmental area (VTA) and DA levels in reward-related brain regions, particularly the nucleus accumbens (NAc). However, the possible functional relationship between hippocampal CBIR transmission and VTA DA neuronal activity is not currently understood. In this study, using in vivo neuronal recordings in rats, we demonstrate that activation of CBIR in the VHipp strongly increases VTA DA neuronal firing and bursting activity, while simultaneously decreasing the activity of VTA non-DA neurons. Furthermore, using a conditioned place preference procedure and a social interaction test, we report that intra-vHipp CBIR activation potentiates the reward salience of normally sub-threshold conditioning doses of opiates and induces deficits in natural sociability and social recognition behaviors. Finally, these behavioral effects were prevented by directly blocking NAc DAergic transmission. Collectively, these findings identify hippocampal CBIR transmission as a critical modulator of the mesolimbic DA pathway and in the processing of reward and social-related behavioral phenomena.

Neuropsychopharmacology (20I5) 40, I436-1447; doi:I0.I038/npp.20 I4.329; published online I4 January 2015
\end{abstract}

\section{INTRODUCTION}

Considerable evidence demonstrates that functional and structural abnormalities in the mammalian ventral hippocampus (vHipp) are critical neuropathological features underlying emotional processing deficits associated with schizophrenia (Grace, 2010; Tseng et al, 2009). Such abnormalities have been reported in the hippocampal regions of schizophrenia patients (Haukvik et al, 2014; Gothelf et al, 2000), and neurodevelopmental studies in rodents attempting to model the behavioral features of schizophrenia have demonstrated that neurons in the vHipp can serve to overdrive the mesolimbic dopamine (DA) system that may, in turn, distort the emotional salience of incoming contextual and sensory information (Grace, 2010; Lodge and Grace, 2007). Nevertheless, the precise neuropharmacological mechanisms within the vHipp that may be

*Correspondence: Dr SR Laviolette, Department of Anatomy and Cell Biology, The Schulich School of Medicine \& Dentistry, University of Western Ontario, 468 Medical Science Building, London, ON N6A 5CI, Canada, Tel: + 5196612111 (extn 80302), Fax: 5196613936 , E-mail: steven.laviolette@schulich.uwo.ca

Received 17 September 20।4; revised 22 November 20।4; accepted 9 December 2014; accepted article preview online 16 December 2014 responsible for the modulation of the mesolimbic DAergic system are not presently understood.

Schizophrenia represents a multifaceted disorder associated with various comorbid features linked to dysregulated motivational and emotional processing, such as elevated rates of substance abuse, including marijuana and opiate-class drugs (Batel, 2000; Cantor-Graae et al, 2001). Disturbances in social cognition and behaviors are also common (Brunet-Gouet and Decety, 2006; Pinkham et al, 2008). Evidence from both human imaging and animal modeling studies suggests that endocannabinoid-mediated modulation of mesocorticolimbic DA transmission may represent a convergent link between vHipp-related functional abnormalities and schizophrenia-related emotional dysregulation (Bossong et al, 2009; Chambers et al, 2001; Tan et al, 2014). Thus, disturbances in cannabinoid type 1 receptor (CB1R) signaling have been linked to the emotional dysregulation characterizing depression and schizophrenia (Burns, 2013). Furthermore, cannabis use during adolescence is considered a risk factor for the later development of psychosis; believed to be associated with overstimulation of the mesolimbic DAergic system (Renard et al, 2014). As a result, there is a growing interest in clarifying the relationship between cannabinoid CB1 receptor 
transmission and DA-mediated emotional and cognitive impairments observed in various psychiatric diseases.

Administration of $\Delta^{9}$-tetrahydrocannabinol (THC), the main psychoactive component of marijuana, or WIN55,212-2, a synthetic CB1R agonist, increases DA levels in both primates and rodents (Justinova et al, 2013). This CB1mediated increase in DA concentrations appears to be important in reward-related brain areas, particularly in the shell subregion of the nucleus accumbens (NAShell), and is thought to result from an increase in the firing frequency of DA neurons located in the ventral tegmental area (VTA) (Oleson and Cheer, 2012). Mesolimbic DAergic activity can be driven by corticolimbic regions, such as the prefrontal cortex (PFC) or the hippocampus (Grace, 2010; Melis et al, 2004), the two structures critically involved in cognitive functions and in which CB1Rs are highly expressed (Marsicano and Lutz, 1999). Indeed, we have reported recently that CB1R activation directly within the PFC can biphasically and dose-dependently increase or decrease the spontaneous activity of downstream DA neurons in the VTA, with a concomitant potentiation or blunting of emotional memory processing correlated with increased or decreased DAergic neuronal activity, respectively (Draycott et al, 2014).

In hippocampus, CB1Rs are mainly found in the presynaptic terminals of inhibitory interneurons (Takács et al, 2014). Activation of these hippocampal CB1Rs has been shown to reduce inhibitory GABAergic tone in both humans and rats, thereby increasing the activity of hippocampal principal neurons (Hájos and Freund, 2002). Furthermore, via excitatory connections to the NAShell, the hippocampus can modulate DA neuronal activity. For example, activation of the vHipp increases both the number of spontaneously active intra-VTA DA neurons (Floresco et al, 2001) and the release of intra-NAShell DA (Legault et al, 2000). These vHipp-mediated effects on DA transmission are hypothesized to be a core feature of schizophrenia pathophysiology (Grace, 2010).

In this study, using an integrative combination of in vivo electrophysiological recordings and behavioral pharmacology in rats, we examined the effects of direct, intra-vHipp CB1R activation on neuronal activity parameters in VTA neuronal subpopulations. In addition, we examined how intra-vHipp modulation of mesolimbic activity may alter reward processing and social behavior and cognition. We demonstrate for the first time that direct activation of CB1R in the vHipp strongly increases VTA DA neuronal activity, potentiates the reward salience of normally nonmotivationally salient conditioning doses of opiates, and induces profound social behavior and memory deficits. These behavioral effects were dependent upon DAergic transmission directly in the NAShell, demonstrating a functional interplay between intra-vHipp cannabinoid transmission and DAergic regulation of affective and social behavioral processing.

\section{MATERIALS AND METHODS}

\section{Animals and Surgical Procedures}

Male Sprague-Dawley rats (300-350 g; Charles River) were used in compliance with the regulations approved by the
Canadian Council for Animal Care and the University of Western Ontario Animal Care and Use committee. Rats were housed under controlled conditions $\left(22-23^{\circ} \mathrm{C}\right.$, a $12-\mathrm{h}$ light/dark cycle with lights on at 0700 , and food and water provided ad libitum). Cannula implantations were performed 10 days before the start of behavioral procedures. Rats were anesthetized with a mixture of ketamine $(80 \mathrm{mg} /$ $\mathrm{ml})$ and xylazine $(6 \mathrm{mg} / \mathrm{ml})$ and placed in a stereotaxic device. Stainless steel guide cannulas (22 gauge, PlasticsOne) were implanted bilaterally into the vHipp at the following coordinates: AP: $-5.6 \mathrm{~mm}$ from bregma, L: $\pm 5.0 \mathrm{~mm}$, DV: $-6.8 \mathrm{~mm}$ from the dural surface. For bilateral cannula implantations in the NAShell, the following coordinates were used: AP: $+1.8 \mathrm{~mm}$ from bregma $\left(12^{\circ}\right.$ angle); $\mathrm{L}: \pm 2.6 \mathrm{~mm}$; DV: $-7.4 \mathrm{~mm}$ from the dural surface. Guide cannulas were held in place using jeweler's screws and dental acrylic cement. After completion of behavioral experiments, rats received an overdose of pentobarbital $(240 \mathrm{mg} / \mathrm{kg}$, i.p., Euthanyl) and were transcardially perfused with isotonic saline followed by $10 \%$ formalin. Brains were extracted and postfixed $24 \mathrm{~h}$ before being placed in a $25 \%$ formalin-sucrose solution for 1 week. Brains were sliced $(40 \mu \mathrm{m})$ using a cryostat and stained with Cresyl violet. Injector tip placements were localized using a light microscope. Rats with cannula placements found outside the anatomical boundaries of the vHipp or NAShell as defined by Paxinos and Watson (2007) were excluded from data analysis.

\section{Drug Administration}

The following drugs were used during behavioral or electrophysiological experiments: the CB1R agonist WIN55,212-2 (WIN55; 0.09 or $0.95 \mathrm{nmol}$; Tocris Bioscience), the selective CB1R antagonist SR141716A (SRA; $0.95 \mathrm{nmol}$; Tocris Bioscience), and the broad-spectrum $\mathrm{DA}_{1-5}$ receptor antagonist, $\alpha$-flupenthixol ( $\alpha$-flu; $1.97 \mathrm{nmol}$; Tocris Bioscience). Both WIN55 and SRA were dissolved in DMSO and then diluted in PBS for a final 1\% DMSO in PBS vehicle solution, whereas $\alpha$-flu was dissolved in PBS $(\mathrm{pH}=7.4)$.

Intra-vHipp microinfusions were performed immediately before each conditioning session or the start of the social interaction test. A total volume of $0.5 \mu \mathrm{l}$ per side was delivered via a 28-gauge microinfusion injector over a period of $1 \mathrm{~min}$. Microinjectors were left in place for an additional $1 \mathrm{~min}$ following drug infusion to ensure adequate diffusion from the tip. Intra-NAShell microinfusions of $\alpha$-flu were performed 3 min before intra-vHipp microinfusions, with a volume of $0.5 \mu \mathrm{l}$ per side.

\section{Conditioned Place Preference}

A total of 88 rats were conditioned using an unbiased conditioned place preference procedure, as described previously (Lintas et al, 2012). Briefly, saline vehicle or morphine injections (i.p.) were paired with one of two environments that differed in color, texture, and smell. As reported previously, rats display no baseline preference for either of these two environments (Laviolette and van der Kooy, 2003). Animals received four morphine-environment and four saline-environment conditioning sessions (1 session/day) and exposure to environments was fully counterbalanced in all experiments. We used a subreward threshold dose of 
morphine $(0.05 \mathrm{mg} / \mathrm{kg}$ i.p. $)$ that we have demonstrated previously to produce no significant place preferences (Lintas et al, 2012). For all experiments, animals received drug or vehicle injections immediately before being placed in either saline- or morphine-paired environments during the conditioning protocol. Each conditioning session was for $30 \mathrm{~min}$. At 1 week after the end of conditioning, rats were tested (drug free) for their place preference during a 10 -min test phase. During testing, rats were placed on a narrow, neutral gray zone that separates the two test compartments and the times spent in each environment (saline- or drug-paired sides; in seconds) were recorded and computerized using a video-tracking system (ANY-maze; Stoelting). CPP behavior was expressed using a place preference score (ie, difference between the time spent in the morphine and saline compartments).

\section{Sociability and Social Recognition Testing}

Social behavior and memory tests were adapted from established protocols (Moy et al, 2004). Testing was performed in a rectangular, three-chambered box (Figure 4). Tested rats $(n=80)$ were first placed in the middle chamber and allowed to explore for $5 \mathrm{~min}$. During this session, doorways into the two side chambers were closed by plastic guillotine doors. Following habituation, an unfamiliar male rat that had no prior contact with the subject rat was placed in one of the side chambers. The location of stranger rat in the left $v s$ right side chamber was counterbalanced between trials. The stranger rat was enclosed in a small rectangular wire cage that allowed nose contact between the bars. Both guillotine doors to the side chambers were then unblocked and the subject was allowed to explore the entire social test apparatus for an 8-min session. An entry was defined as all four paws in one chamber. Times spent in each chamber were recorded and analyzed by a video-tracking system (ANY-maze; Stoelting). Behavioral performances were expressed using sociability scores (ie, difference between the time spent in the stranger and empty compartments). Rats showing no preference for the stranger box obtained sociability scores equal to zero (chance level), whereas rats showing preferences for the stranger box obtained sociability scores significantly higher than zero. At the end of this sociability test, each rat was immediately tested in a second 8-min session to evaluate their social recognition abilities. A second, unfamiliar rat was placed in the chamber that had been empty during the first 8 -min session. This second stranger was also enclosed in an identical small wire cage. The tested rat had a choice between the first, alreadyinvestigated unfamiliar rat and the novel unfamiliar rat. In this situation, control rats spend significantly more time with the new stranger, demonstrating a natural preference for social novelty. Measures were taken of the amount of time spent in each chamber and a social recognition score (ie, difference between the time spent in the nonfamiliar rat and familiar rat chamber) was attributed to each tested rat.

\section{VTA Neuronal Activity Recordings and Analysis}

In vivo single-cell extracellular recordings in the VTA were performed as described previously (Draycott et al,
2014). Briefly, 53 rats were anesthetized with urethane $(1.4 \mathrm{~g} / \mathrm{kg}$, i.p.) and placed in a stereotaxic frame with body temperature maintained at $37^{\circ} \mathrm{C}$. A scalp incision was made and a hole was drilled in the skull overlaying the vHipp and the VTA. For intra-vHipp microinfusions of WIN55 or SRA, a $1 \mu$ l Hamilton syringe was slowly lowered into the vHipp using the same stereotaxic coordinates described above. For intra-VTA recordings, glass microelectrodes (with an average impedance of $6-8 \mathrm{M} \Omega$ ) filled with a $2 \%$ pontamine sky blue solution were lowered using a hydraulic micropositioner (Kopf640) and at the following coordinates: AP: $-4.9 \mathrm{~mm}$ from bregma, $\mathrm{L}: \pm 0.7 \mathrm{~mm}, \mathrm{DV}:-7.0$ to $-8.5 \mathrm{~mm}$ from the dural surface. Extracellular signals were amplified using a MultiClamp700B amplifier (Molecular Devices) and recorded through a Digidata1440A acquisition system (Molecular Devices) using pClamp10 software. Recordings were filtered at $1 \mathrm{kHz}$ and sampled at $5 \mathrm{kHz}$. VTA DA neurons were identified according to wellestablished electrophysiological feature (Grace and Bunney, 1983): (1) a relatively long action potential width ( $>2.5 \mathrm{~ms}$ ), (2) a slow spontaneous firing rate $(2-5 \mathrm{~Hz}),(3)$ a triphasic waveform consisting of a notch on the rising phase followed by a delayed after potential, and (4) a single irregular or bursting firing pattern. In contrast, VTA non-DAergic cells were characterized based upon previously reported criteria: (1) a narrow action potential width ( $<1 \mathrm{~ms}),(2)$ a biphasic waveform, (3) relatively fast firing rates $(10-20 \mathrm{~Hz})$, and (4) the absence of burst.

Electrophysiological analyses were performed using the Clampfit10 (Molecular Devices) software package. The response patterns of isolated VTA neurons to microinfusion of cannabinoid compounds into the vHipp were determined by comparing the neuronal frequency rates between the 5 min preinfusion versus postinfusion recording epochs. Classification of drug infusion effects used a criterion of a $\geq 10 \%$ increase in firing frequency after infusion to be classified as an 'increase' effect, and a $\leq 10 \%$ decrease to be classified as a 'decrease' effect. Neurons showing firing frequency parameters within these cutoff points were classified as 'no change'. In VTA DA neurons, we also analyzed bursting rate (number of burst events per min) and the number of spike events within a burst. The onset of a burst was defined as the occurrence of two consecutive spikes with an interspike interval of $<80 \mathrm{~ms}$. For histological analysis of extracellular VTA neuronal recording sites, recording electrodes positions were marked with an iontophoretic deposit of pontamine sky blue dye $(-20 \mu \mathrm{A}$, continuous current for $15 \mathrm{~min}$ ). Brain extraction and slicing were similar to those described for cannula placement verifications. Sections were stained with neutral red and neuronal recording sites were confirmed with light microscopy. Cells recorded outside the anatomical boundaries of the VTA, as defined by Paxinos and Watson (2007), were excluded from data analysis.

\section{Statistical Analyses}

Behavioral and electrophysiological data were analyzed with a one-way ANOVA or Student's $t$-tests where appropriate. The post hoc analyses were performed with Fisher's LSD or Newman-Keuls tests. 


\section{RESULTS}

\section{Effects of intra-vHipp CB1R Activation on VTA DA Neuronal Activity}

To determine whether CB1R activation in the vHipp modulates VTA DA neuron activity, we performed singlecell extracellular recordings in urethane-anesthetized animals (see Materials and Methods). Recording sites in the VTA and microinfusion locations in the vHipp are shown in Figure 1a. We sampled a total of $n=65$ VTA DA neurons (Vehicle group, $n=10$ cells in 9 rats; WIN55 $50 \mathrm{ng} / 0.5 \mu \mathrm{l}$ group, $n=15$ cells in 11 rats; WIN55 $500 \mathrm{ng} / 0.5 \mu \mathrm{l}$ group, $n=15$ cells in 12 rats; and WIN55 (500 ng/0.5 $\mu \mathrm{l})+$ SRA $(500 \mathrm{ng} / 0.5 \mu \mathrm{l})$ group, $n=15$ cells in 12 rats; SRA $(500 \mathrm{ng} / 0.5 \mu \mathrm{l})$ group, $n=10$ cells in 10 rats $)$. In rats receiving intra-vHipp vehicle microinfusion, $70 \%$ of neurons did not change their firing frequency, $10 \%$ of neurons showed an increase in firing activity, and $20 \%$ demonstrated a decrease in activity levels. For rats receiving the lower dose of WIN55, 60\% of neurons increased their firing frequency, $20 \%$ of neurons showed a decrease in firing activity, and $20 \%$ demonstrated no change in activity levels. Intra-vHipp WIN55 (500 ng) gave the same increased tendency. Indeed, $73.3 \%$ of neurons increased their firing frequency, $6.7 \%$ decreased their firing activity, and $20 \%$ demonstrated no change in activity levels. In contrast, only $26.7 \%$ of neurons increased firing frequency following microinfusion of WIN55 + SRA; $60 \%$ did not change their activity and $13.3 \%$ decreased firing frequency. Finally, following intra-vHipp microinfusion of SRA alone, $40 \%$ of the recorded cells decreased their firing frequency, $20 \%$ of neurons increased their firing activity, and $40 \%$ demonstrated no change in activity levels. Examples of the main firing frequency effects according to each treatment are represented in Figure 1f, g, and h. Analyses of neuronal firing frequencies revealed average changes from baseline of $-8 \%$ for rats treated with vehicle, $+28 \%$ for rats treated with WIN55 (50 ng), + 56\% with WIN55 (500 ng), + 7\% for rats receiving WIN55 + SRA, and $-10 \%$ for rats microinfused with SRA (500 ng). Statistical analyses showed that the firing frequency changes obtained with intra-vHipp WIN55 (50 ng) and WIN55 (500 ng) were significantly different from preinfusion baseline levels $\left(t_{14}=2.585\right.$, $p<0.05 ; t_{14}=4.632, p<0.001$, respectively), whereas the changes following intra-vHipp vehicle, WIN55 + SRA, and SRA were not significantly different from baseline $(p>0.05)$. ANOVA revealed a significant treatment effect $\left(\mathrm{F}_{2,64}=8.276, p<0.01\right)$ and post hoc comparisons showed that rats with intra-vHipp WIN55 (500 ng) had an average VTA DA neuronal firing frequency significantly higher than rats microinfused with the vehicle solution $(p<0.001)$, the lower dose of WIN55 $(p<0.05)$, the WIN55 + SRA solution $(p<0.001)$, and with SRA $(p<0.001)$. Analysis of bursting rate revealed average changes from baseline of $-5,+19$, $+53,-2$, and $-17 \%$ for rats treated with intra-vHipp vehicle, WIN55 (50 ng), WIN55 (500 ng), WIN55 + SRA, and SRA (500 ng), respectively (Figure 1d). Statistical analyses showed that bursting rates following intra-vHipp WIN55 (50 ng) and WIN55 (500 ng) were significantly elevated relative to baseline levels $\left(t_{14}=2.791, p<0.05 ; t_{14}=3.694\right.$, $p<0.001$, respectively), whereas bursting rates following intra-vHipp vehicle, WIN55+SRA, and SRA were not significantly different from baseline levels $(p>0.05)$. ANOVA comparing bursting rates before $v s$ after infusion revealed a significant effect of treatment $\left(\mathrm{F}_{2,64}=6.772\right.$, $p<0.001$ ), and post hoc comparisons showed that bursting rates were significantly increased following intra-vHipp WIN55 (500 ng) relative to rats treated with the vehicle solution $(p<0.01)$, the lower dose of WIN55 $(p<0.05)$, the $\mathrm{WIN}+$ SRA solution $(p<0.001)$, and the SRA treatment $(p<0.001)$. Analyses of the number of spikes per burst event revealed average changes from baseline of $-5 \%$ with the vehicle solution, $+21 \%$ with WIN55 (50 ng), $+45 \%$ with WIN55 (500 ng), + 10\% with WIN + SRA, and $+2 \%$ with SRA (500 ng) (Figure 1e). Statistical analyses showed that the number of spikes per burst following intra-vHipp WIN55 (500 ng) was significantly increased relative to preinfusion baseline $\left(t_{14}=4.544, p<0.001\right)$. However, neither rats treated with a lower dose of WIN55 (50 ng) nor receiving co-administration with the $\mathrm{CB} 1$ antagonist (WIN55 + SRA) showed significant changes relative to baseline ( $p$ 's $>0.05)$. ANOVA revealed no significant effect of treatment $\left(\mathrm{F}_{2,64}=2.499, p>0.05\right)$ on number of spikes per burst event before $v s$ after infusion; however, post hoc analyses showed that the average number of spikes per burst event following intra-vHipp WIN55 (500 ng) was significantly higher relative to rats receiving intra-vHipp vehicle solution $(p<0.01)$, WIN55 + SRA $(p<0.05)$, and SRA $(500 \mathrm{ng})(p<0.05)$. Thus, intra-vHipp CB1R activation dose-dependently increases VTA DA neuronal firing frequency and bursting activity through a pharmacologically specific CB1R-dependent substrate.

\section{Effects of Intra-vHipp CB1R Activation on VTA Non-DA Neuronal Activity}

Non-DA neurons isolated in the VTA were also recorded and analyzed. We sampled a total of $n=56$ cells (Vehicle group, $n=10$ cells in 9 rats; WIN55 $50 \mathrm{ng} / 0.5 \mu$ l group, $n=13$ cells in 11 rats; WIN55 $500 \mathrm{ng} / 0.5 \mu$ l group, $n=13$ cells in 12 rats; WIN55 (500 ng/ $0.5 \mu \mathrm{l})+$ SRA $(500 \mathrm{ng} / 0.5 \mu \mathrm{l})$ group, $n=10$ cells in 12 rats; and SRA $500 \mathrm{ng} / 0.5 \mu \mathrm{l}, n=10$ cells in 10 rats). In rats receiving intra-vHipp vehicle microinfusion, $60 \%$ of neurons did not change their firing frequency, $30 \%$ of neurons showed an increase in firing activity, and $10 \%$ demonstrated a decrease in activity levels. For rats receiving the lower dose of WIN55, 38.5\% of the recorded cells demonstrated decreased firing frequency, $15.4 \%$ showed increased firing, and $46.1 \%$ demonstrated no change (Figure 2a). For rats receiving the higher dose of WIN55, $53.8 \%$ of neurons decreased firing rates, $30.8 \%$ showed increased firing, and $15.4 \%$ demonstrated no change. For rats receiving the coinfusion of WIN55 (500 ng) and SRA (500 ng), 30\% of neurons decreased their firing frequency, $40 \%$ increased their activity levels, and $30 \%$ showed no change in firing activity. Finally, following intra-vHipp microinfusion of SRA alone, $42.9 \%$ of the recorded cells did not change their firing frequency, $42.9 \%$ of neurons increased their firing activity, and $14.2 \%$ demonstrated increase in activity levels. Examples of the main firing frequency effects according to each treatment are represented in Figure 1c, d, and e. Analyses of the firing frequencies revealed average changes relative to preinfusion baselines of $+9 \%$ in the vehicle group, $-12 \%$ for rats 
a

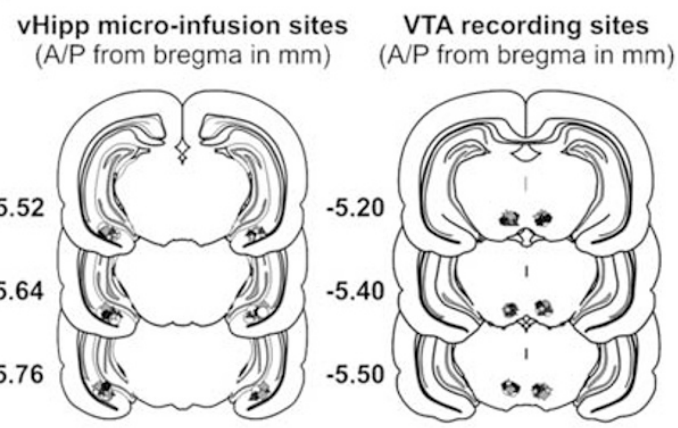

b

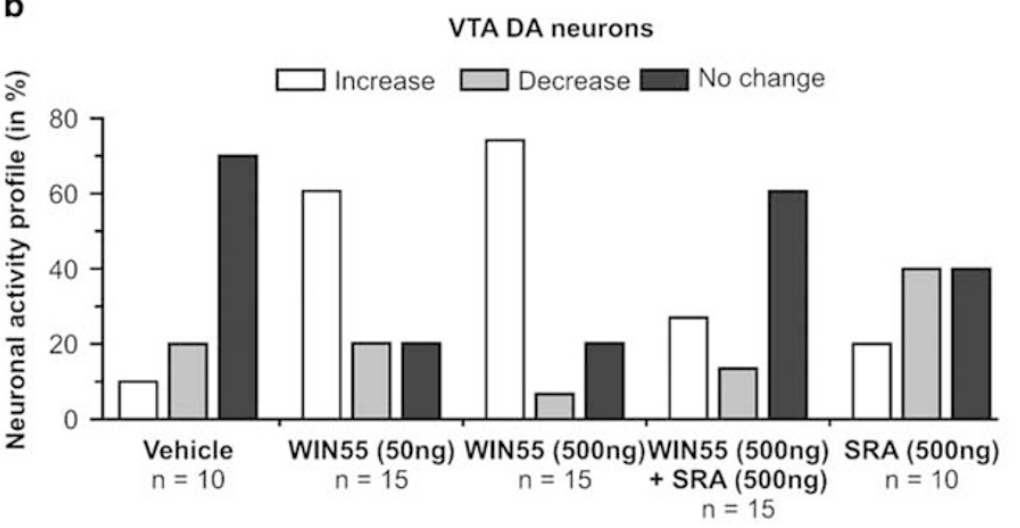

C

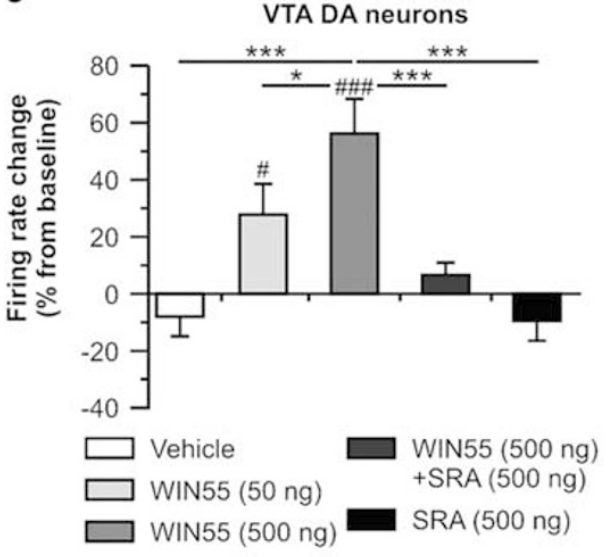

f
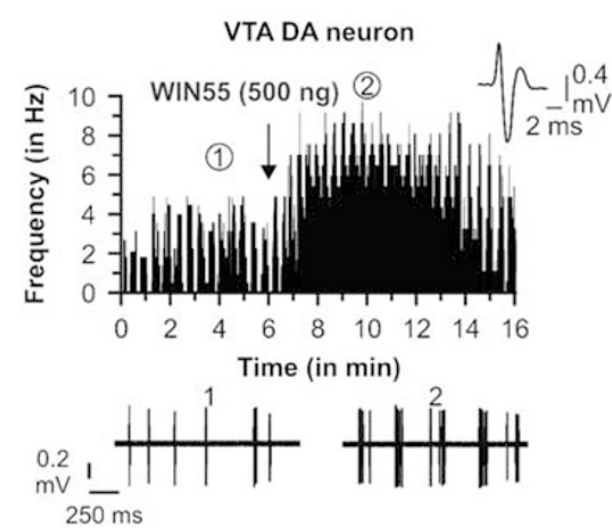

d

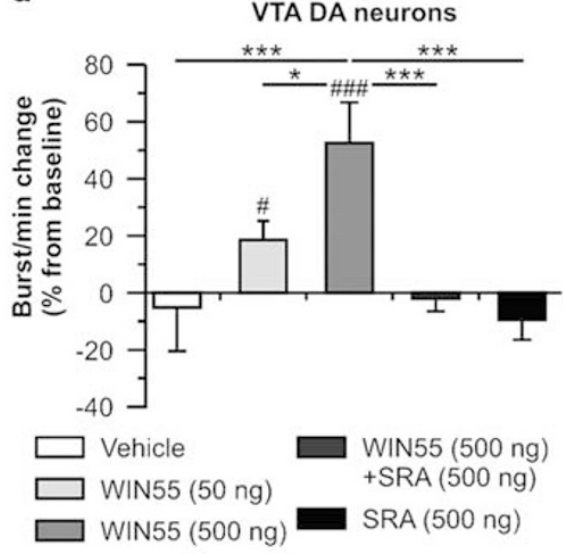

g
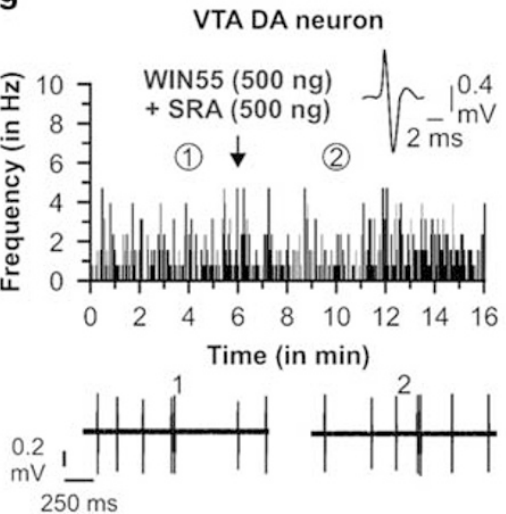

e VTA DA neurons

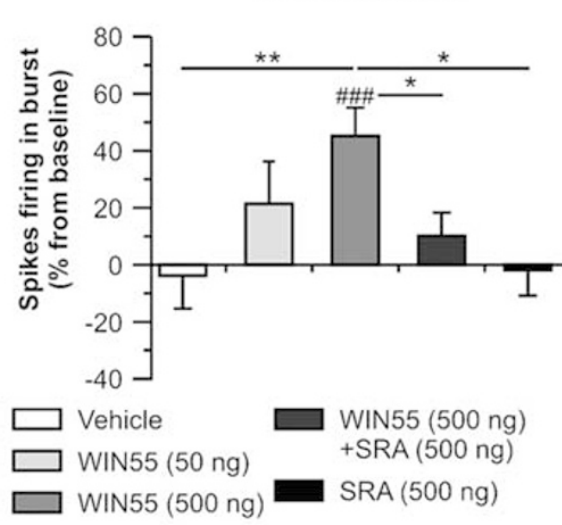

h
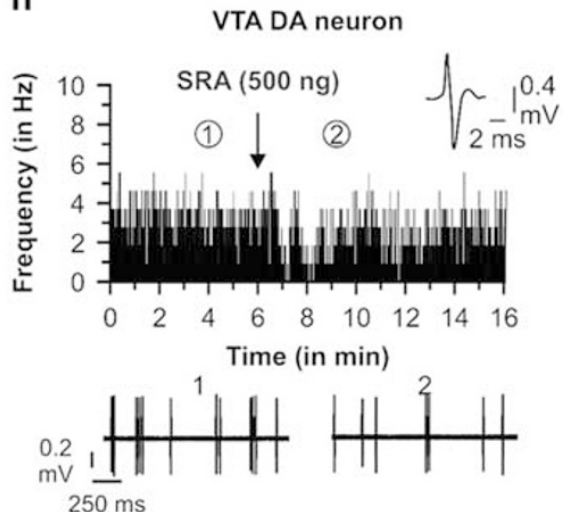

Figure I Effects of intra-vHipp WIN55, WIN55 + SRA, and SRA microinfusions on VTA DA neuronal activity. (a) Histological localization of microinfusion sites in the vHipp and recording sites in the VTA for each treatment condition performed during electrophysiological recordings (stars: vehicle; diamonds: WIN55 (50 ng); circles: WIN55 (500 ng); triangles: WIN55 + SRA; and pentagone: SRA (500 ng)). WIN55 and SRA doses were given in a total volume of $0.5 \mu \mathrm{l}$. (b) Summary of the VTA DA neuronal activity profile (ie, number of cells that increased, decreased, or did not change their firing frequency after microinfusions). (c) Consequences of intra-vHipp vehicle, WIN55 (50 ng and $500 \mathrm{ng}$ ), WIN55 + SRA, and SRA (500 ng) treatments on VTA DA neuronal firing frequency, (d) burst rate and (e) spikes firing in burst $\left(* p<0.05, * * p<0.0\right.$ I and ${ }^{*} * * * 0<0.00$ I vs other treatment; ${ }^{*} p<0.05,{ }^{*} p<0.0$ I and $\# \#$ \# $<0.00$ I vs baseline, ie, 0 ). (f) Representative histogram showing the increase in response activity of one DA neuron following microinfusion of the higher dose of WIN55. (g) Example of a DA neuron showing no change in activity level following coinfusion of WIN55 + SRA. (h) Example of a DA neuron showing a nonsignificant decrease in firing frequency after intra-vHipp microinfusion of SRA (500 ng). For each panel, inset shows the action potential waveform of the selected neuron; (I) and (2) show the activity patterns recorded before (baseline activity) and after the microinfusions respectively.

treated with WIN55 (50 ng), 23\% with WIN55 (500 ng), $+4 \%$ for rats receiving WIN55 + SRA, and $+4 \%$ in rats microinfused with SRA (500 ng) (Figure 2b). Statistical comparison between average firing frequency changes and baseline levels showed that intra-vHipp WIN55 (500 ng) significantly decreased firing levels $\left(t_{12}=-2.801, p<0.05\right)$, whereas changes observed with the vehicle, WIN55 (50 ng), WIN55 + SRA, and SRA (500 ng) solutions were not significant ( $p$ 's $>0.05)$. ANOVA revealed a significant effect of the treatment factor $\left(\mathrm{F}_{2,55}=3.079, p<0.5\right)$. The post hoc 


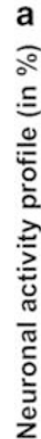

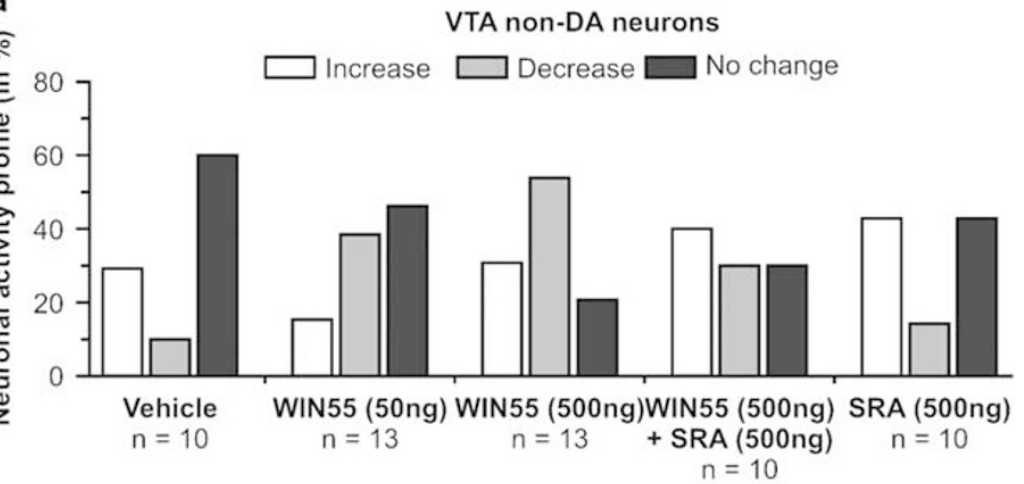

c

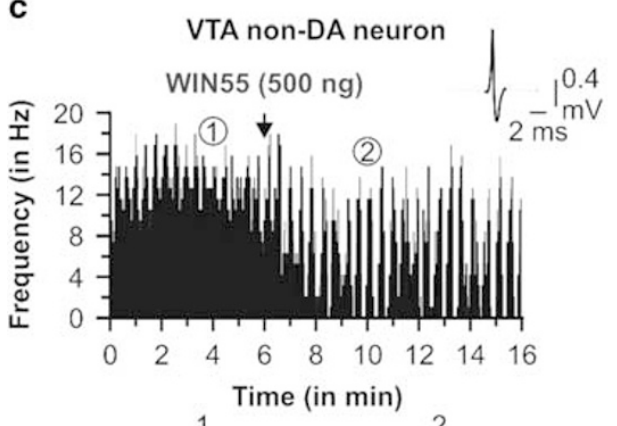

1 d
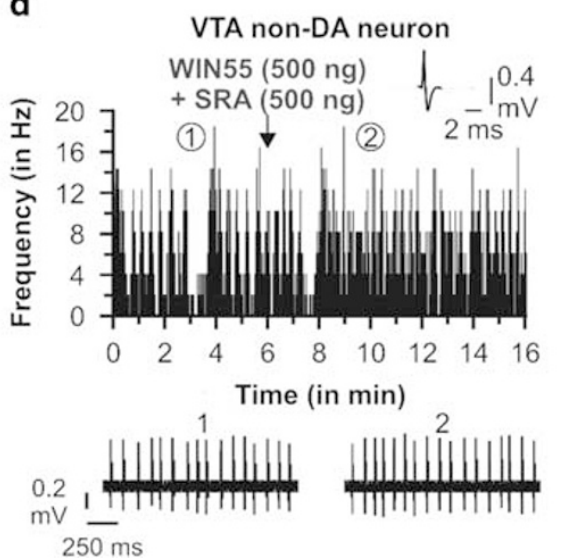

b

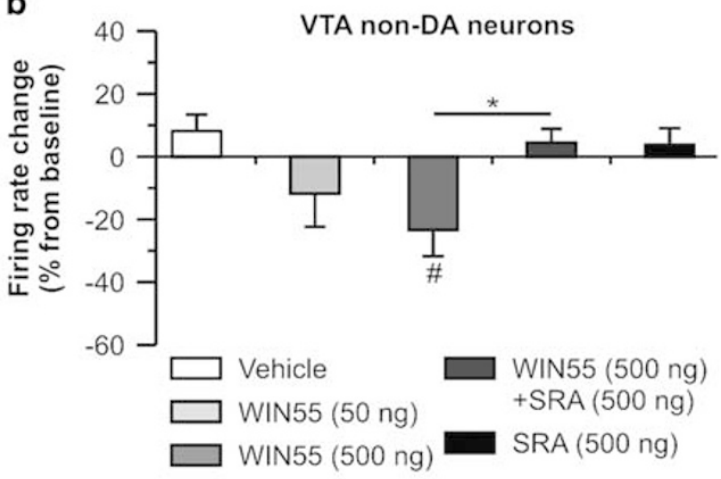

e

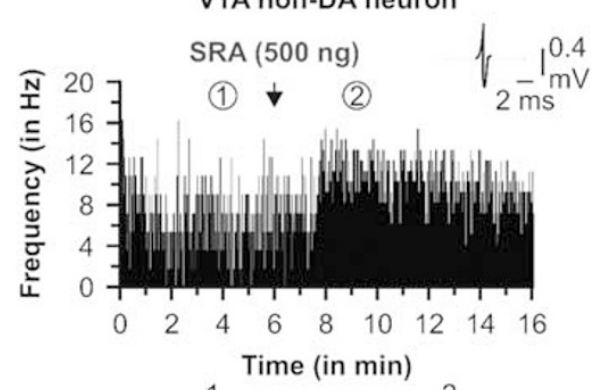

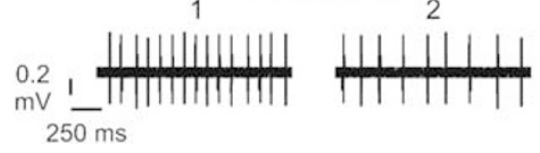

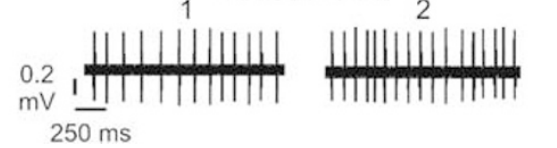

Figure 2 Effects of intra-vHipp WIN55, WIN55 + SRA, and SRA microinfusions on VTA non-DA (putative GABA interneurons) neuronal activity. (a) Summary of the VTA non-DA neuronal activity profile (ie, number of cells that increased, decreased, or did not change their firing frequency after microinfusions). WIN55 and SRA doses were given in a total volume of $0.5 \mu \mathrm{l}$. (b) Consequences of intra-vHipp vehicle, WIN55 (50 ng and $500 \mathrm{ng}$ ), WIN55 + SRA, and SRA (500 ng) treatments on VTA GABA neuronal firing frequency $\left(* p<0.05\right.$ vs other treatment; ${ }^{*} p<0.05$, vs baseline, ie, 0$)$. (c) Representative histogram showing the decrease in firing activity of a single VTA non-DA neuron following microinfusion of the higher dose of WIN55. (d) One VTA non-DA neuron showing no change in activity profile following microinfusion of WIN55 + SRA. (e) Example of a non-DA neuron showing a nonsignificant increase in firing activity after intra-vHipp microinfusion of SRA ( $500 \mathrm{ng}$ ). For each panel, inset shows an action potential waveform of the represented neuron; (I) and (2) show the activity patterns recorded before (baseline activity) and after the microinfusions respectively.

comparisons showed that rats treated with intra-vHipp WIN55 (500 ng) had an average VTA non-DA neuronal firing rate significantly lower than rats receiving vehicle $(p<0.01)$, WIN55 + SRA $(p<0.05)$, or SRA solution $(p<0.05)$. Thus, intra-vHipp CB1R activation significantly decreased the spontaneous firing rates of non-DA neurons.

\section{Effects of Intra-vHipp CB1R Activation on Morphine Reward Salience}

We next assessed the potential role of vHipp CB1R in modulating opiate reward salience by examining the effects of intra-vHipp WIN55 on the acquisition of a normally subreward threshold conditioning dose of morphine $(0.05 \mathrm{mg} / \mathrm{kg}$ i.p.) using an unbiased CPP procedure (see Materials and Methods; Figure 3a). Concentrations and group sizes after histological verifications were as follows: vehicle $(n=9)$, WIN55 (50 ng, $n=7)$, WIN55 (500 ng, $n=8)$, WIN55 + SRA $(n=7)$, and SRA (500 ng, $n=8)$. Results of CPP testing are presented in Figure $3 \mathrm{~b}$. ANOVA analysis of CPP test scores revealed a significant effect of treatment $\left(\mathrm{F}_{4,38}=5.293, p<0.01\right)$ on times spent in morphine- $v s$ vehicle-paired environments. The post hoc analyses showed that rats microinfused with the higher dose of WIN55 (500 ng) demonstrated a significant CPP for morphine-paired environments relative to vehicle-treated controls, rats receiving a lower dose of WIN55 $(50 \mathrm{ng})$, rats microinfused with WIN55+SRA, or with SRA alone ( $p$ 's $<0.05$ ). To control for any possible motivational effects of intra-vHipp WIN55 in and of itself, we tested the ability of the highest behaviorally effective dose of intra-vHipp WIN55 (500 ng, $n=9$ ) compared with intra-vHipp PBS infusion $(n=9)$ in the same place conditioning procedure (Figure 3c and d). Statistical analyses revealed no difference between the two groups $\left(t_{16}=0.116, p>0.05\right)$ or between PPS and the chance level (for both treatments, $p>0.05$ ). Thus, intra-vHipp CB1R activation dose-dependently potentiates the reward salience of normally subreward threshold doses of morphine through a pharmacologically specific CB1R-dependent substrate while producing no motivational effects in and of itself.

\section{Effects of Intra-vHipp CB1R Activation on Social Interaction and Social Memory}

Disturbances in both DAergic and cannabinoid signaling are associated with social behavior/memory disturbances 
a

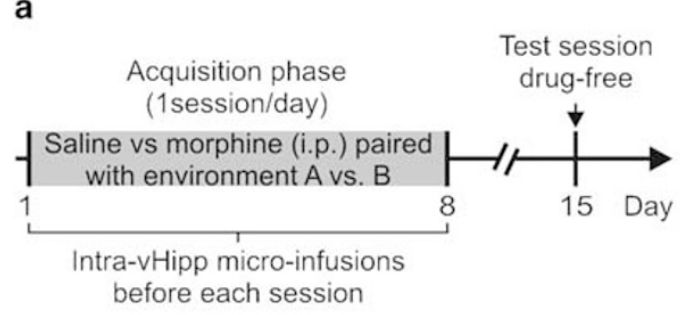

b

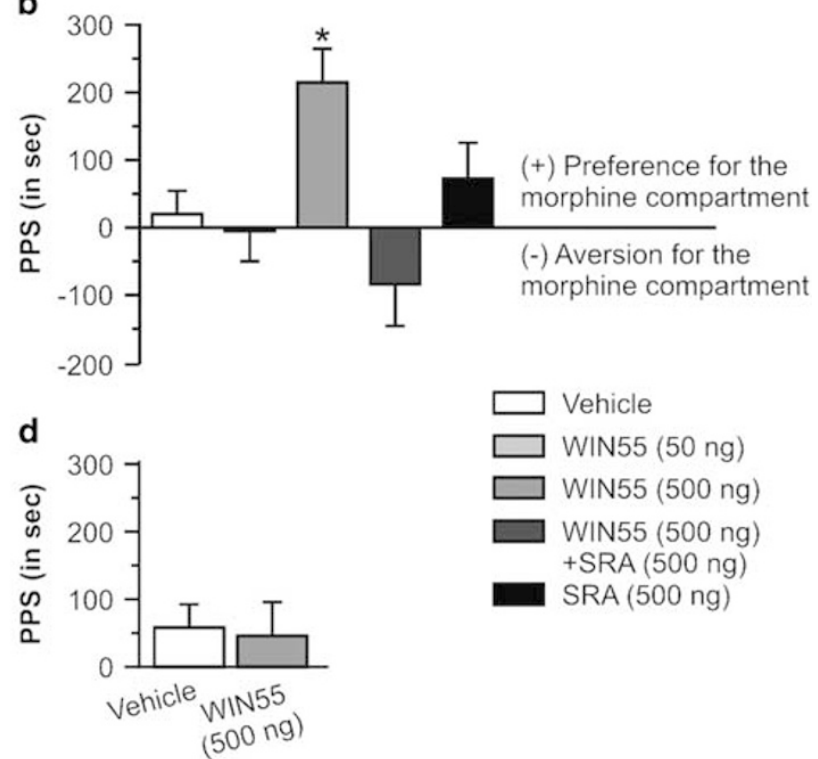

Figure 3 Effects of intra-vHipp WIN55, WIN55 + SRA, and SRA on conditioned place preference using a subreward threshold dose of morphine. (a) Conditioned place preference protocol, WIN55 was bilaterally microinfused into the vHipp before each acquisition session. WIN55 and SRA doses were given in a total volume of $0.5 \mu \mathrm{l}$ per hemisphere. (b) Place preference scores (PPS: difference between the time spent in the morphine- and saline-paired compartments). $* P<0.05$ vs the three other groups. (c) We further tested whether intra-vHipp WIN55 (500 ng vs vehicle) was able to produce a place preference by itself. (d) Note the absence of place preference when intra-vHipp WIN55 was paired with one of the two environments during acquisition. Graphs represent mean + SEM.

a
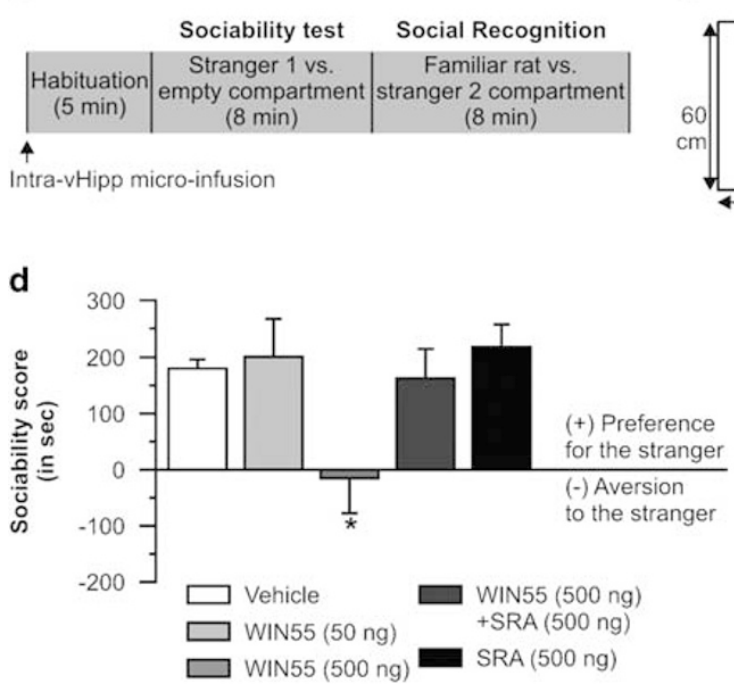

b
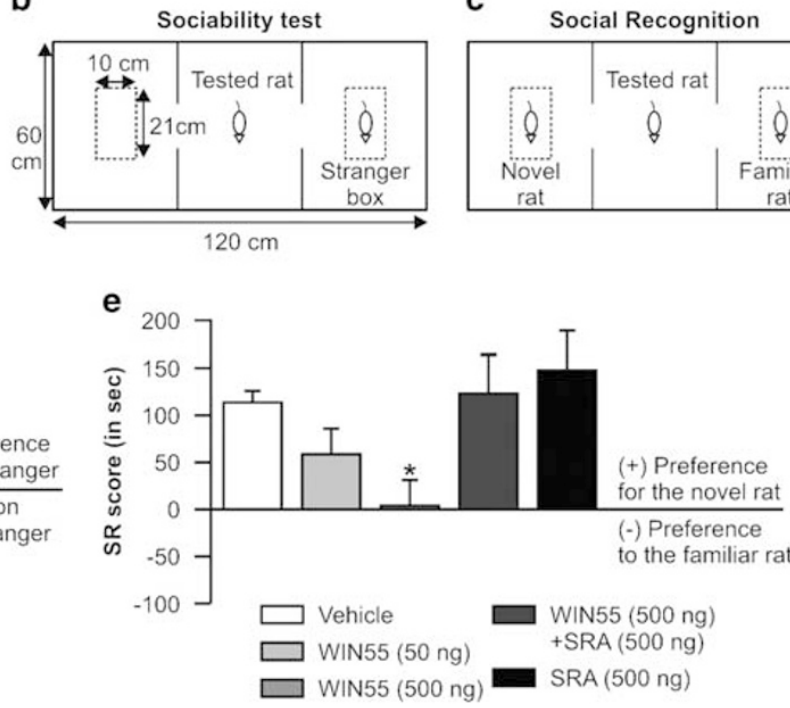

Figure 4 Effects of intra-vHipp WIN55, WIN55 + SRA, and SRA on sociability and social recognition. (a) The social interaction test started with a 5-min habituation session followed by two consecutive 8-min interaction sessions (ie, sociability test and social recognition test). Intra-vHipp microinfusions were performed immediately before the habituation session. WIN55 and SRA doses were given in a total volume of $0.5 \mu$ l per hemisphere. (b, c) Apparatus and rats' placements for the sociability and social recognition test respectively. (d) Sociability scores. Note the absence of preference for the stranger compartment in rats treated with intra-vHipp WIN55 (500 ng). (e) Social recognition (SR) scores. Graphs represent mean + SEM. * $P<0.05$ vs the three other groups.

(Brunet-Gouet and Decety, 2006; Pinkham et al, 2008). Therefore, we next examined the potential effects of intravHipp CB1 receptor activation on rats' natural sociability and social recognition (Figure 4a). The experimental protocols for these experiments are summarized in
Figure $4 \mathrm{~b}$ and $\mathrm{c}$. Concentrations and group sizes after histological verifications of cannula placement were as follows: vehicle $(n=9)$, WIN55 (50 ng, $n=8)$, WIN55 (500 ng, $n=9)$, WIN55 + SRA $(n=10)$, and SRA (500 ng, $n=8$ ). For the sociability score (Figure 4d), ANOVA 


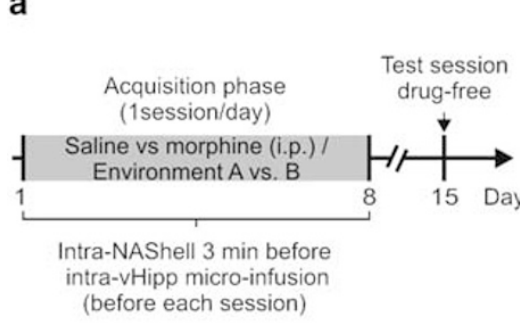

b

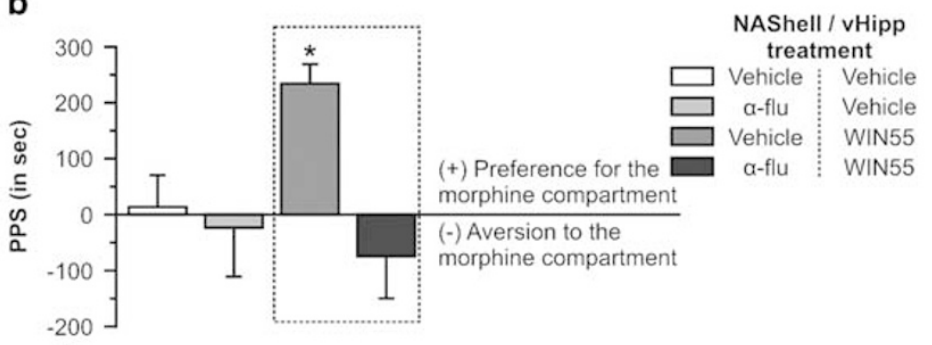

C

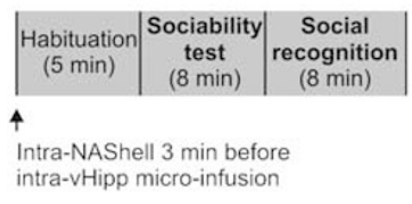

d

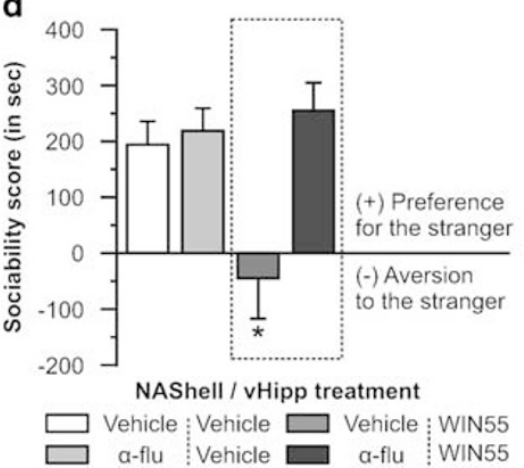

e

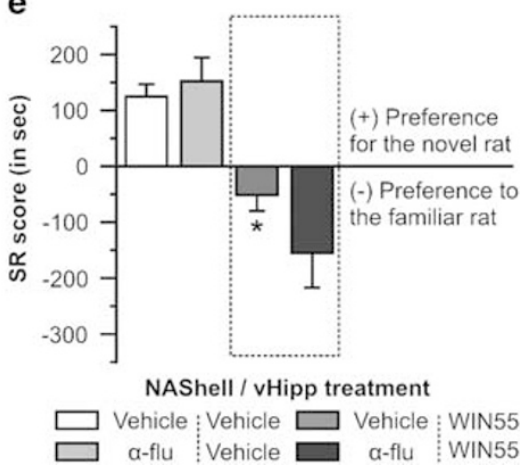

Figure 5 Effects of intra-NAShell DA receptors blockade on morphine CPP and social interaction alterations induced by intra-vHipp WIN55 (500 ng/ $0.5 \mu \mathrm{l})$. (a) Conditioned place preference protocol. Intra-NAShell microinfusions of $\alpha$-flupenthixol $(\alpha$-flu, I $\mu \mathrm{g} / 0.5 \mu \mathrm{l})$ were performed 3 min before intravHipp microinfusions. (b) Place preference scores. Note that intra-NAShell microinfusion of $\alpha$-flu prevented the place preference for a subreward threshold dose of morphine induced by intra-vHipp WIN55 (dashed rectangle; ${ }^{*} p<0.05$ vs chance level, ie, 0; ${ }^{*} p<0.05$ vs the three other groups). (c) Sociability and social recognition timeline. Intra-NAShell microinfusions were performed 3 min before intra-vHipp microinfusions. (d) Sociability scores and (e) social recognition (SR) scores. Note that intra-NAShell microinfusion of $\alpha$-flu was able to prevent the sociability deficit induced by intra-vHipp WIN55 but not the social recognition alteration (dashed rectangles). $* P<0.05$ vs the three other groups. Graphs represent mean + SEM.

analyses revealed a significant effect of the treatment factor $\left(\mathrm{F}_{4,43}=2.710, p<0.05\right)$ on sociability scores and post hoc analyses showed that rats receiving the higher dose of WIN55 obtained significantly lower sociability scores relative to the other experimental groups $(p<0.05)$. For the social recognition test (Figure 4e), ANOVA analysis of social recognition scores revealed a significant effect of treatment $\left(\mathrm{F}_{4,43}=3.173, p<0.05\right)$ on times spent with novel $v s$ familiar rats, and post hoc comparisons showed that rats treated with intra-vHipp WIN55 (500 ng) obtained social recognition scores significantly lower than vehicle control or rats receiving a lower dose of WIN55 $(p<0.05)$. Thus, intra-vHipp CB1R activation dose-dependently attenuates natural sociability and social recognition memory through a pharmacologically specific CB1R substrate.

\section{NAShell DA Receptor Transmission Is Required for vHipp CB1R-Mediated Modulation of Reward and Social Behavior Processing}

Given our previous effects demonstrating that behaviorally effective doses of intra-vHipp WIN55 were sufficient to increase firing and bursting levels of VTA DAergic neurons, we next tested whether bilateral intra-NAShell microinfusions of the DA receptor antagonist, $\alpha$-flu $(1 \mu \mathrm{g} / 0.5 \mu \mathrm{l})$ would modulate the behavioral effects we obtained with the higher dose of WIN55 $(500 \mathrm{ng} / 0.5 \mu \mathrm{l})$. For the place preference test (Figure $5 \mathrm{a}$ and $\mathrm{b}$ ), group sizes after histological verifications of cannulas placement were as follows (NAShell/vHipp treatment): vehicle/vehicle $(n=7)$, vehicle/WIN55 $(n=7), \alpha$ flu/vehicle $(n=7)$, and $\alpha$-flu/WIN55 $(n=7)$. ANOVA of CPP test scores showed a significant effect of treatment $\left(\mathrm{F}_{3,27}=4.067, p<0.05\right)$ on times spent in vehicle $v s$ morphine environments. The post hoc analyses revealed that rats receiving intra-NAShell vehicle before WIN55 microinfusions displayed a significantly greater CPP relative to vehicle/vehicle, vehicle/ $\alpha$-flu, or $\alpha$-flu/WIN55 control groups $(p$ 's $<0.05)$. For the sociability test (Figure $5 c$ and d), group sizes were as follows: vehicle/vehicle $(n=7)$, vehicle/WIN55 $(n=7), \alpha$-flu/vehicle $(n=7)$, and $\alpha$-flu/ WIN55 $(n=7)$. ANOVA revealed a significant effect of treatment on times spent with novel $v s$ familiar rats $\left(\mathrm{F}_{3,27}=6.649, p<0.01\right)$. The post hoc analysis showed that rats microinfused with vehicle/WIN55 demonstrated sociability scores significantly lower than vehicle/vehicle, vehicle/ $\alpha$-flu, or $\alpha$-flu/WIN55 control groups $(p<0.05)$. For the social recognition test (Figure $5 \mathrm{e}$ ), ANOVA analysis of social recognition scores revealed a significant effect of treatment on times spent with novel $v s$ familiar rats $\left(\mathrm{F}_{3,27}=6.649\right.$, $p<0.01)$. The post hoc comparisons demonstrated that rats receiving vehicle/WIN55 treatment obtained an average social recognition score significantly lower than vehicle/ vehicle, vehicle/ $\alpha$-flu, or $\alpha$-flu/WIN55 control groups $(p<0.05)$. Thus, blockade of DA transmission directly in the NAShell was sufficient to prevent the effects of intravHipp CB1R activation on both opiate reward salience modulation and disruption of sociability.

\section{DISCUSSION}

Cannabinoid signaling can potently regulate the salience of emotional information and associative memory formation 
by modulating both reward or aversion-related memory processing (Ahmad et al, 2013; Laviolette and Grace, 2006; Tan et al, 2014). Characterizing the underlying neural circuitry responsible for these effects may provide new insights into how dysregulation of the endocannabinoid system may lead to disturbances in emotional processing characteristic of schizophrenia. In this study, we found that direct activation of vHipp CB1Rs increased VTA DAergic neuronal activity in terms of spontaneous firing and bursting rates, while concomitantly decreasing the activity of non-DAergic neurons. Behaviorally, intra-vHipp CB1R activation amplified the associative rewarding effects of morphine and induced deficits in natural sociability and social recognition behaviors. Finally, we demonstrated that these behavioral modifications were dependent on NAShell DA receptor transmission.

Dysregulation of the mesocorticolimbic DA system is a predominant theory of schizophrenia, proposed to account for the observed disturbances in both emotional and social cognition. However, the neurobiological mechanisms underlying this pathological DAergic state are still a source of debate. Nevertheless, converging evidence from both human and animal modeling studies support the hypothesis that hyperactivity in the ventral hippocampus (anterior hippocampus in humans) might overdrive the DA system in schizophrenia, leading to disrupted gating of emotionally salient information (Grace, 2010). For example, excitatory chemical stimulation of the rat vHipp increases both the number of spontaneously active VTA DAergic neurons (Floresco et al, 2001) and the release of DA in the NAShell (Legault et al, 2000), a brain region known to be involved in the detection of motivationally salient information.

One potential mechanism underlying hyperactive hippocampal $>$ DA drive may involve a loss of GABAergic inhibitory control of hippocampal principal neurons projecting to mesolimbic structures. Indeed, post-mortem studies have shown decreased density of parvalbuminpositive GABA interneurons in the hippocampus of schizophrenia patients (Zhang and Reynolds, 2002). More importantly, activation of $\mathrm{CB} 1$ receptors within the human hippocampus has been reported to strongly inhibit the release of GABA from inhibitory networks (Katona et al, 2000). Moreover, this loss of inhibition in the hippocampal circuitry has been characterized in an animal model reproducing schizophrenia-like pathophysiology, including increased DAergic tone (Lodge et al, 2009). Interestingly, CB1Rs are highly expressed in CCK-positive interneurons, a second subpopulation of hippocampal GABA interneurons (Takács et al, 2014). Activation of these CB1Rs is known to reduce $\mathrm{GABA}$ release, thereby increasing the activity of hippocampal excitatory principal neurons (Hájos and Freund, 2002). Using in vivo single-cell electrophysiological recordings, we found that intra-vHipp microinfusion of a CB1R agonist significantly increased both firing frequency and bursting rates of VTA DA neurons. The coinfusion of the selective CB1R antagonist SR141716A completely blocked the effects induced by WIN55 on DA neuronal activity, pointing to the specific involvement of the vHipp $\mathrm{CB} 1 \mathrm{R}$ in these effects. Finally, the microinfusion of SR141716A alone in the vHipp did not significantly change the neuronal firing activity of VTA DA cells.
The influence exerted by the vHipp on VTA DA neuronal population activity is not direct, as there are no monosynaptic anatomical connections from the vHipp to the VTA. As such, it is likely that the modulation of DA cell activity by the vHipp occurs via a polysynaptic circuit (Luo et al, 2011). For example, Floresco et al (2001) demonstrated that excitatory connections originating from the vHipp and connected directly to the NAc are critically involved in the control exerted by the vHipp on VTA DA neuronal activity. Indeed, intra-NAc microinfusion of the glutamate receptor antagonist kynurenic acid prevented the increase of spontaneously active DA cells following vHipp stimulation. Moreover, the NAc can influence DA neuronal activity by both an indirect and a direct projection to the VTA. For example, there are major GABAergic projections from the NAc to the ventral pallidum and activation of the NAc can block the tonic inhibitory influence exerted by the ventral pallidum on VTA DA cells (Floresco et al, 2003). In addition, recent evidence employing an optogenetic approach indicates that NAc GABAergic medium spiny neurons project densely to VTA non-DA neurons (Xia et al, 2011). VTA non-DA neurons comprise glutamate cells and GABAergic local interneurons. Thus, if the NAc-connected non-DA neurons are GABAergic neurons that make local contacts with DA neurons (Omelchenko and Sesack, 2009), activation of this projection would result in an increase of VTA DA neuronal firing. In this study, we found that activation of vHipp CB1R significantly decreased the firing activity of non-DA cells. Thus, one possibility is that the observed effects on VTA DA neuron activity patterns are mediated via the inhibition of VTA GABAergic interneurons following activation of VTA-projecting NAc medium spiny neurons. Another potential anatomical pathway that could give rise to an increase in activity of DAergic cells involves the rostromedial tegmental nucleus (RMTg), also named the tail of the ventral tegmental area (Bourdy and Barrot, 2012). Indeed, the RMTg receives inhibitory inputs from the NAc and sends dense GABAergic projections to VTA DA neurons. Activation of the NAc to RMTg pathway would result in VTA DA neuronal disinhibition. Interestingly, it has been recently demonstrated that the decrease of GABA release in the VTA induced by activation of $\mu$-opioid receptors occurs predominantly at RMTg GABAergic inputs to VTA DA neurons (Matsui et al, 2014). In this case, our results showing an increase in activity of VTA DA neurons following activation of CB1R in the vHipp would predict a potentiation of the psychopharmacological properties of opiate compounds.

Recent advances in the study of DA cells have begun to define the diversity of functional phenotypes among midbrain DA neurons depending on DA cell distinct projections and their differential afferent inputs, as well as assigning specific behavioral functions to each subpopulation of DA cells (Roeper, 2013). For example, optogenetic phasic stimulation of the laterodorsal tegmentum, which preferentially excited NAc-projecting DA VTA neurons, induced a robust conditioned place preference, whereas optogenetic phasic stimulation of the lateral habenula, which excited PFC-projecting DA VTA neurons, induced significant conditioned place aversions (Lammel et al, 2011). Although in our electrophysiological experiments the majority of the VTA DA neurons increased their firing 
activity after intra-vHipp microinfusion of WIN55, 10 to $20 \%$ of DA neurons decreased their activity and $20 \%$ of the recorded DA neurons did not respond to WIN55. This heterogeneity of neuronal response to vHipp CB1R agonist might reflect DA VTA cells diversity of inputs. For example, NAc GABAergic neurons send direct projections to both DA and GABA neurons in the VTA (Matsui et al, 2014). Thus, activation of the vHipp to NAc pathways might result in not only an increase in activity of DA neurons via different disinhibition mechanisms, but also a decrease in firing activity in some subpopulations of VTA DA neurons. It is also important to note that the vHipp sends excitatory connections to the medial prefrontal cortex (mPFC) and that $\mathrm{mPFC}$ pyramidal neurons send descending glutamatergic projections to both DA and GABAergic VTA cells (Wu et al, 2013). Therefore, the diversity of VTA DA activity profile we obtained following intra-vHipp WIN55 was possibly because of the activation of different pathways including the vHipp-mPFC and/or vHipp-NAc circuits.

Changes in the activity pattern of DA cells from tonic to burst pattern firing has been associated with phasic DA release in the NAShell, a signal proposed to occur when an organism is presented with an emotionally salient stimulus (Bromberg-Martin et al, 2010). Our results suggest that vHipp CB1R activation may induce a hyperactive DAergic activity state. In this case, in the presence of intrahippocampal CB1R stimulation, any arriving stimulus would be predicted to overdrive mesolimbic DA-dependent processing of emotional salience, leading to distortions in emotional sensory filtering. Indeed, such effects are present following overactivation of CB1R substrates in both the PFC and basolateral nucleus of the amygdala, whereby normally sub-threshold fear-related stimuli become highly salient associative cues (Laviolette and Grace, 2006; Tan et al, 2014). In addition, the acute effects of cannabis, even in otherwise healthy individuals, include deficits in accurate in emotional salience filtering and emotional regulation (Gruber et al, 2009; Metrik et al, 2013). The presence of dysregulated motivational salience has been suggested to be a common mechanism underlying psychosis and an increased use of illicit drugs, including opiates, characterized in schizophrenia (Batel, 2000). In this study, we found that microinfusion of a nonrewarding dose of WIN55 into the vHipp, during the acquisition phase of a morphine CPP protocol, was able to potentiate the rewarding properties of a normally nonsalient conditioning dose of systemic morphine (Ahmad et al, 2013). This effect was blocked by coadministration of a selective CB1R antagonist and with direct blockade of DA receptor transmission within the NAShell. Our results showing the ability of vHipp CB1R activation to produce a place preference for an ineffective dose of morphine has also been demonstrated using subreward threshold doses of alcohol (Rezayof et al, 2012). However, the involvement of the DAergic system in the interaction between hippocampal cannabinoid transmission and ethanol remains to be determined.

Dysregulation of emotional processing may not only lead to misattribution of motivational salience to environmental cues, but also disrupt the processing of stimuli that would otherwise be behaviorally significant, such as social interaction behaviors and cognition. Notably, the occurrences of antisocial and depressive personality traits represent a high-risk subgroup of schizophrenia patients vulnerable to drugs of abuse (Batel, 2000). Moreover, increasing evidence suggests that a common single neural circuit may be involved in processing the motivational salience of both social and nonsocial events, with the ventral striatum playing a critical functional role (Ruff and Fehr, 2014). We found that intra-vHipp CB1R activation induced deficits in normal social behavior, including attenuation in sociability behaviors and impaired social recognition memory. These behavioral perturbations were blocked by coinfusion of the selective CB1R antagonist SR141716A. Moreover, the blocking of DA receptors within the NAc prevented the social deficit induced by intra-vHipp CB1R activation. Interestingly, during the social recognition test, the DA receptor antagonist and WIN55 treatment induced a strong preference for the familiar rat. In other words, this group of rats remembered the first stranger and spent more time with it instead of showing a preference for social novelty. Modification of DA brain levels can affect cognitive flexibility, a phenomenon well described in obsessive and compulsive disorders (Klanker et al, 2013) and also in schizophrenia (Floresco et al, 2009). For example, inhibition of D2/D3 receptor subtypes in primates results in difficulties in adapting behavior following changing task demands, but not during acquisition of the original discrimination (Lee et al, 2007). Therefore, in this study, the combination of DA receptor blockade and vHipp CB1R activation on DA transmission might have led to cognitive inflexibility expressed by a preference for a familiarity than novelty.

In summary, our study provides evidence that $\mathrm{CB} 1 \mathrm{R}$ transmission within the vHipp can powerfully modulate mesolimbic DAergic activity. Furthermore, activation of the CB1R receptor substrates within the vHipp serves to modulate both reward-related processing and normal social interactions and cognition. Given the well-established involvement of both CB1R and vHipp-related dysregulation in the pathogenesis of schizophrenia-related behavioral abnormalities, the present findings implicate a role for CB1 signaling directly within the vHipp as a potential mechanism underlying hippocampal-mediated overdrive of the mesolimbic DA pathway.

\section{FUNDING AND DISCLOSURE}

The authors declare no conflict of interest.

\section{ACKNOWLEDGEMENTS}

This work was supported by the Canadian Institutes of Health Research (CIHR; MOP 246144) and the National Science and Engineering Research Council of Canada (NSERC).

\section{REFERENCES}

Ahmad T, Lauzon NM, Jaeger de X, Laviolette SR (2013). Cannabinoid transmission in the prelimbic cortex bidirectionally controls opiate reward and aversion signaling through dissociable kappa versus $\mu$-opiate receptor dependent mechanisms. J Neurosci 33: 15642-15651.

Batel P (2000). Addiction and schizophrenia. Eur Psychiatry 15: 115-122. 
Bossong MG, Berckel BNM, van Boellaard R, Zuurman L, Schuit RC, Windhorst $\mathrm{AD}$ et al (2009). Delta 9-tetrahydrocannabinol induces dopamine release in the human striatum. Neuropsychopharmacology 34: 759-766.

Bourdy R, Barrot M (2012). A new control center for dopaminergic systems: pulling the VTA by the tail. Trends Neurosci 35: 681-690.

Bromberg-Martin ES, Matsumoto M, Hikosaka O (2010). Dopamine in motivational control: rewarding, aversive, and alerting. Neuron 68: 815-834.

Brunet-Gouet E, Decety J (2006). Social brain dysfunctions in schizophrenia: a review of neuroimaging studies. Psychiatry Res 148: 75-92.

Burns JK (2013). Pathways from cannabis to psychosis: a review of the evidence. Front Psychiatry 4: 1-12.

Cantor-Graae E, Nordström LG, McNeil TF (2001). Substance abuse in schizophrenia: a review of the literature and a study of correlates in Sweden. Schizophr Res 48: 69-82.

Chambers RA, Krystal JH, Self DW (2001). A neurobiological basis for substance abuse comorbidity in schizophrenia. Biol Psychiatry 50: 71-83.

Draycott B, Loureiro M, Ahmad T, Tan H, Zunder J, Laviolette SR (2014). Cannabinoid transmission in the prefrontal cortex biphasically controls emotional memory formation via functional interactions with the ventral tegmental area. J Neurosci 34: 13096-13109.

Floresco SB, Todd CL, Grace A (2001). Glutamatergic afferents from the hippocampus to the nucleus accumbens regulate activity of ventral tegmental area dopamine neurons. J Neurosci 21: 4915-4922.

Floresco SB, West AR, Ash B, Moore H, Grace AA (2003). Afferent modulation of dopamine neuron firing differentially regulates tonic and phasic dopamine transmission. Nat Neurosci 6: 968-973.

Floresco SB, Zhang Y, Enomoto T (2009). Neural circuits subserving behavioral flexibility and their relevance to schizophrenia. Behav Brain Res 204: 396-409.

Gothelf D, Soreni N, Nachman RP, Tyano S, Hiss Y, Reiner O et al (2000). Evidence for the involvement of the hippocampus in the pathophysiology of schizophrenia. Eur Neuropsychopharmacol 10: $389-395$.

Grace A (2010). Ventral hippocampus, interneurons, and schizophrenia: a new understanding of the pathophysiology of schizophrenia and its implications for treatment and prevention. Curr Dir Psychol Sci 19: 232-237.

Grace AA, Bunney BS (1983). Intracellular and extracellular electrophysiology of nigral dopaminergic neurons - 1. Identification and characterization. Neuroscience 10: 301-315.

Gruber SA, Rogowska J, Yurgelun-Todd DA (2009). Altered affective response in marijuana smokers: an FMRI study. Drug Alcohol Depend 105: 139-153.

Hájos N, Freund F (2002). Distinct cannabinoid sensiti v e receptors regulate hippocampal excitation and inhibition. Chemi Phys Lipids 121: 73-82.

Haukvik UK, Westlye LT, Mørch-Johnsen L, Jørgensen KN, Lange EH, Dale AM et al (2014). In vivo hippocampal subfield volumes in schizophrenia and bipolar disorder. Biol Psychiatry (e-pub ahead of print 3 July 2014; doi:10.1016/j.biopsych.2014.06.020).

Justinova Z, Mascia P, Wu H-Q, Secci ME, Redhi GH, Panlilio L et al (2013). Reducing cannabinoid abuse and preventing relapse by enhancing endogenous brain levels of kynurenic acid. Nat Neurosci 16: 1652-1661.

Katona I, Sperlágh B, Maglóczky Z, Sántha E, Köfalvi A, Czirjak S et al (2000). GABAergic interneurons are the targets of cannabinoid actions in the human hippocampus. Neuroscience 100: 797-804.

Klanker M, Feenstra M, Denys D (2013). Dopaminergic control of cognitive flexibility in humans and animals. Front Neurosci 7: 201.
Lammel S, Ion DI, Roeper J, Malenka RC (2011). Projectionspecific modulation of dopamine neuron synapses by aversive and rewarding stimuli. Neuron 70: 855-862.

Laviolette SR, Grace A (2006). The roles of cannabinoid and dopamine receptor systems in neural emotional learning circuits: implications for schizophrenia and addiction. Cell Mol Life Sci 63: 1597-1613.

Laviolette SR, van der Kooy D (2003). Blockade of mesolimbic dopamine transmission dramatically increases sensitivity to the rewarding effects of nicotine in the ventral tegmental area. $\mathrm{Mol}$ Psychiatry 8: 50-59.

Lee B, Groman S, London ED, Jentsch JD (2007). Dopamine D2/D3 receptors play a specific role in the reversal of a learned visual discrimination in monkeys. Neuropsychopharmacology 32: 2125-2134.

Legault M, Rompré PP, Wise RA (2000). Chemical stimulation of the ventral hippocampus elevates nucleus accumbens dopamine by activating dopaminergic neurons of the ventral tegmental area. J Neurosci 20: 1635-1642.

Lintas A, Chi N, Lauzon NM, Bishop SF, Sun N, Tan H et al (2012). Inputs from the basolateral amygdala to the nucleus accumbens shell control opiate reward magnitude via differential dopamine D1 or D2 receptor transmission. Eur J Neurosci 35: 279-290.

Lodge D, Grace A (2007). Aberrant hippocampal activity underlies the dopamine dysregulation in an animal model of schizophrenia. J Neurosci 27: 11424-11430.

Lodge DJ, Behrens MM, Grace AA (2009). A loss of parvalbumincontaining interneurons is associated with diminished oscillatory activity in an animal model of schizophrenia. J Neurosci 29: 2344-2354.

Luo AH, Tahsili-Fahadan P, Wise RA, Lupica CR, Aston-Jones G (2011). Linking context with reward: a functional circuit from hippocampal CA3 to ventral tegmental area. Science 333: 353-357.

Marsicano G, Lutz B (1999). Expression of the cannabinoid receptor $\mathrm{CB} 1$ in distinct neuronal subpopulations in the adult mouse forebrain. Eur J Neurosci 11: 4213-4225.

Matsui A, Jarvie BC, Robinson BG, Hentges ST, Williams JT (2014). Separate GABA afferents to dopamine neurons mediate acute action of opioids, development of tolerance, and expression of withdrawal. Neuron 82: 1346-1356.

Melis M, Perra S, Muntoni AL, Pillolla G, Lutz B, Marsicano G et al (2004). Prefrontal cortex stimulation induces 2-arachidonoylglycerol-mediated suppression of excitation in dopamine neurons. J Neurosci 24: 10707-10715.

Metrik J, Kahler CW, Mcgeary JE, Monti PM, Rohsenow DJ (2013). Acute effects of marijuana smoking on negative and positive affect. J Cogn Psychother 25: 1-19.

Moy SS, Nadler JJ, Perez A (2004). Sociability and preference for social novelty in five inbred strains: an approach to assess autistic-like behavior in mice. Genes Brain Behav 3: 287-302.

Oleson EB, Cheer JF (2012). A brain on cannabinoids: the role of dopamine release in reward seeking. Cold Spring Harb Perspect Med 2: 1-13.

Omelchenko N, Sesack SR (2009). Ultrastructural analysis of local collaterals of rat ventral tegmental area neurons: GABA phenotype and synapses onto dopamine and GABA cells. Synapse 63: 895-906.

Paxinos G, Watson C (2007). The Rat Brain Atlas in Stereotaxic Coordinates. Academic Press: San Diego.

Pinkham AE, Hopfinger JB, Pelphrey KA, Piven J, Penn DL (2008). Neural bases for impaired social cognition in schizophrenia and autism spectrum disorders. Schizophr Res 99: 164-175.

Renard J, Krebs M-O, Pen Le G, Jay TM (2014). Long-term consequences of adolescent cannabinoid exposure in adult psychopathology. Front Neurosci 8: 1-14.

Rezayof A, Ghandipour M, Nazari-Serenjeh F (2012). Effect of co-injection of arachydonilcyclopropylamide and ethanol on conditioned place preference in rats. Physiol Behav 107: 301-308. 
Roeper J (2013). Dissecting the diversity of midbrain dopamine neurons. Trends Neurosci 36: 336-342.

Ruff CC, Fehr E (2014). The neurobiology of rewards and values in social decision making. Nat Rev Neurosci 15: 549-562.

Takács VT, Szőnyi A, Freund TF, Nyiri G, Gulyás AI (2014). Quantitative ultrastructural analysis of basket and axo-axonic cell terminals in the mouse hippocampus. Brain Struct Funct (e-pub ahead of print 10 January 2014; doi:10.1007/s00429-0130692-6).

Tan H, Ahmad T, Loureiro M, Zunder J, Laviolette SR (2014). The role of cannabinoid transmission in emotional memory formation: implications for addiction and schizophrenia. Front Psychiatry 5: 1-12.
Tseng KY, Chambers RA, Lipska BK (2009). The neonatal ventral hippocampal lesion as a heuristic neurodevelopmental model of schizophrenia. Behav Brain Res 204: 295-305.

Wu J, Gao M, Shen J-X, Shi W-X, Oster AM, Gutkin BS (2013). Cortical control of VTA function and influence on nicotine reward. Biochem Pharmacol 86: 1173-1180.

Xia Y, Driscoll JR, Wilbrecht L, Margolis EB, Fields HL, Hjelmstad GO (2011). Nucleus accumbens medium spiny neurons target nondopaminergic neurons in the ventral tegmental area. J Neurosci 31: 7811-7816.

Zhang ZJ, Reynolds GP (2002). A selective decrease in the relative density of parvalbumin-immunoreactive neurons in the hippocampus in schizophrenia. Schizophr Res 55: 1-10. 